\title{
Initial experience with the EndoAssist camera-holding robot in laparoscopic urological surgery
}

\author{
Sashi S. Kommu • Peter Rimington • \\ Christopher Anderson · Abhay Rané
}

Received: 27 October 2006/ Accepted: 8 January 2007 / Published online: 9 March 2007

(C) Springer London 2007

\begin{abstract}
Although the advantages of laparoscopic surgery are well documented, one disadvantage is that, for optimum performance, an experienced camera driver is required who can provide the necessary views for the operating surgeon. In this paper we describe our experience with urological laparoscopic techniques using the novel EndoAssist robotic camera holder and review the current status of alternative devices. A total of 51 urological procedures (25 using the EndoAssist device and 26 using a conventional human camera driver) conducted by three experienced surgeons were studied prospectively, including nephrectomy (simple and radical), pyeloplasty, radical prostatectomy, and radical cystoprostatectomy. The surgeon noted the extent of body comfort and muscle fatigue in each case. Other aspects documented were ease of scope movement, i.e. usability, need to clean the telescope, time of set-up, surgical performance, and whether it was necessary to change the position of the arm during the surgery. All three surgeons involved in the evaluation felt comfortable throughout all procedures, with no loss of autonomy. It was, however, obvious that the large arc generated whilst doing a nephrectomy led to
\end{abstract}

\section{S. S. Kommu}

The Bristol Urological Institute, Bristol, UK

P. Rimington

Eastbourne Hospital, Eastbourne, UK

C. Anderson

St George's Hospital, London, UK

A. Rané $(\square)$

East Surrey Hospital, Canada Avenue,

Redhill, Surrey RH1 1RH, UK

e-mail: a.rane@btinternet.com more episodes of lens cleaning, and the arm had to be relocated on some occasions. Clearer benefits were seen while performing pelvic surgery or pyeloplasty, perhaps because the arc of movement was smaller. The EndoAssist is an effective, easy to use device for robotic camera driving which reduces the constraint of having to have an experienced camera driver for optimum visualisation during laparoscopic urological procedures.

Keywords Laparoscopic surgery - Robot - Surgery · Device $\cdot$ Camera $\cdot$ EndoAssist

\section{Introduction}

Although the advantages of laparoscopic surgery are well documented, there are significant challenges not only to the surgeon but also to the camera driver. In traditional laparoscopic surgery the operating surgeon does not have direct visual control of the operative field. The surgeon depends on the camera assistant to manoeuvre the camera for optimum visualization. This is compounded by the fact that cooperation must occur on a real-time basis with each step. As such, independent driver bias arises where conflicts of cooperation can occur in which the surgeon's optimum view is somewhat hampered by the camera driver's perception of what the optimum view should be. Manual camera control can also be physically demanding leading to fatigue and a suboptimum visual field. During prolonged procedures frustration and conflicts can occur. Very often an experienced camera driver is required who can provide the necessary views for the operating surgeon. Whereas in some units the laparoscopic team 
can afford to use an experienced camera driver, in most units this is not economically feasible on a regular basis. Ideally, the surgeon should have full control of all instruments required that are directly required for conducting a given procedure. This includes surgical operative instruments and control of the operative field.

The purpose of non-human camera holders is to return camera-control to the surgeon and to stabilize the visual field during minimally invasive procedures. As such, active and passive camera holders have been developed in a bid to offer the surgeon an alternative and better tool for control of the operating surgeon's direct visual field. The published advantages include:

1 elimination of the fatigue of the assistant who holds the camera;

2 elimination of fine motor tremor and small inaccurate movements; and

3 delivery of a steady and tremor-free image [1-6].

In this study, we describe our experience with urological laparoscopic techniques using a novel robotic camera holder (EndoAssist; Armstrong Healthcare, High Wycombe, Bucks, UK) (Fig. 1). The EndoAssist is a unique robot that is controlled by simple head movement by the surgeon and enables complete autonomy over camera movement. Movement is executed by a head-mounted infrared emitter; the sensor is

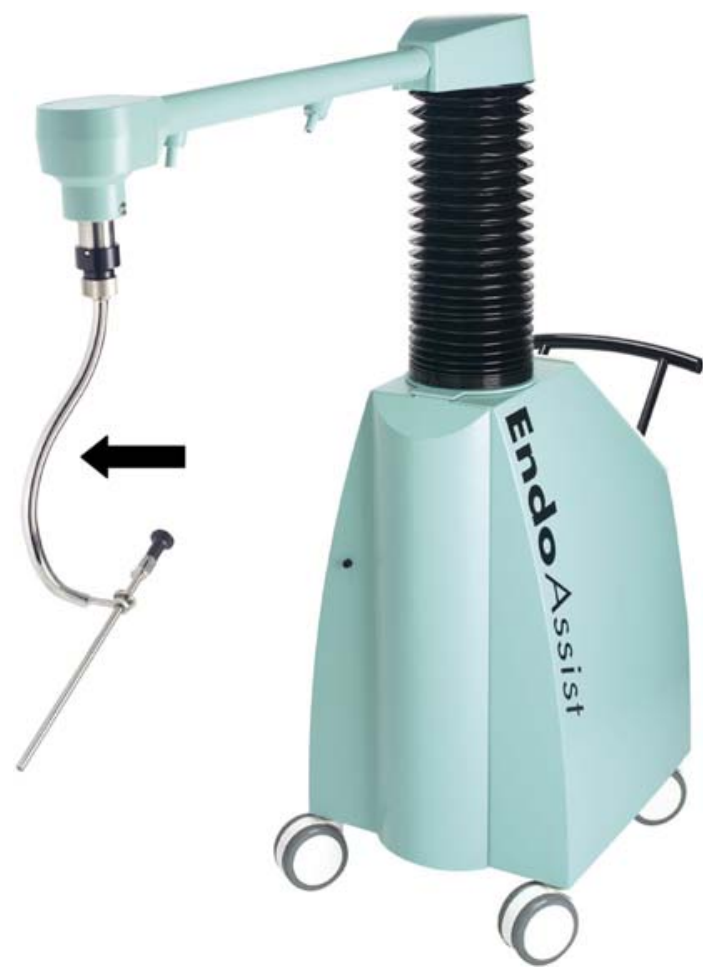

Fig. 1 The arrow shows the camera driver of the EndoAssist placed above the monitor and picks up any operator executed head movements (Fig. 2). The foot clutch ensures there is no unnecessary travel when movement is not required.

A total of 51 urological procedures conducted by three experienced surgeons were studied. The surgeon noted the extent of body comfort and muscle fatigue in each case. Other aspects documented were ease of scope movement, need to clean the telescope, time of set-up, surgical performance, and whether it was necessary to change the position of the arm during the surgery. We also reviewed the current literature.

\section{Materials and methods}

The study included a total of 51 urological procedures in which two arms were used, the Endoassist arm [EArm] and the conventional arm [C-Arm], which involves a human camera holder or driver. For the EndoAssist arm, data were prospectively collected for 25 procedures. For the conventional arm, data for 26 cases were retrospectively collected from our database. The procedures were conducted by three experienced surgeons and included nephrectomy (simple and radical), pyeloplasty, radical prostatectomy, and radical cystoprostatectomy. The surgeon noted:

1 the extent of body comfort and muscle fatigue in each case, by using a modified body part discomfort score (BPDS), a score of 0 implying no discomfort during the procedure and 10 being sufficient discomfort to stop the task before recommencing;

2 ease of scope movement or usability;

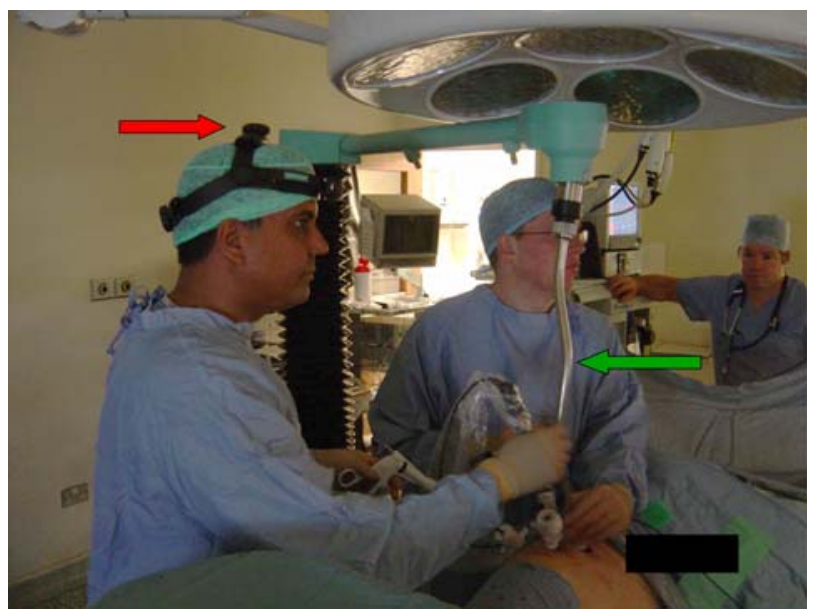

Fig. 2 Head-mounted infra-red emitter (red arrow) and the camera driver being positioned (green arrow) 
3 need to clean the telescope;

4 time of set-up and effect on overall operative time;

5 surgical performance; and

6 whether it was necessary to change the position of the arm during surgery.

Ease of scope movement was graded on basis of the International Organization for Standardization (ISO) which defines usability as the extent to which goals are achieved with effectiveness, efficiency, and satisfaction. Each of these was graded on a linear scale of 1-5, from lowest to highest. The number of times the scope had to be cleaned was also documented for each of the cases for both the E-Arm and C-Arm. Time to set up was also tabulated as mean time in minutes \pm standard deviation. The E-Arm data were collected prospectively whereas the C-Arm data was collected from pooled data that were already available. A thirty-degree laparoscope was used for the renal surgery and a $0^{\circ}$ scope was used for the pelvic surgery. The Harmonic scalpel (Ethicon Endosurgery, Bracknell, UK), the Olympus SonoSurg (Keymed, Southend, UK), or the Lotus (SRA Developments Ashburton, UK) were used to aid circumferential specimen mobilisation. Hemolok (Weck, High Wycombe, Bucks, UK) clips were used as appropriate for securing pedicles. Where statistical analysis was performed in this study, we used a Wilcoxon matched pairs signed-rank test and a result was deemed statistically significant if $P<0.05$. All data were analysed by use of a preformed computer generated template of the variables of interest. This template was developed with a signature unique to each case which could be used for data mining and fusion.

\section{Results}

All cases included in this study were free from major intraoperative complications including major bleeding or other factors which would have demanded additional haemostatic or reconstructive steps.

With regard to the extent of body comfort and muscle fatigue, all three surgeons involved with the evaluation felt comfortable with the E-Arm for each of the procedures studied, with no loss of autonomy. The surgeons were uncomfortable with use of the C-Arm for laparoscopic radical prostatectomy, and prompting for motion adjustment was required repeatedly for the cases studied. There was no reported difference between muscle fatigue for the two modes in the cases analysed. The overall BPDS was 2.1 for the E-Arm and 2.2 for the C-Arm $(P=0.2)$ indicating no statistically significant difference between the two.
With regard to ease of scope movement and the need to clean the telescope, we found that, on average, the large arc generated whilst performing a nephrectomy led to more episodes of lens cleaning for the EArm group than for the C-Arm group. For laparoscopic nephrectomy, furthermore, the EndoAssist port had to be relocated on several occasions whereas the C-Arm group did not require camera port relocation. Fewer problems were encountered while performing pelvic surgery or pyeloplasty, perhaps because the arc of movement was smaller. The grading for ease of scope movement was, on average, 3 for radical prostatectomy, 2 for pyeloplasty, and 1 for laparoscopic nephrectomy. There was a statistically significant difference between ease of scope movement, i.e. "usability", in favour of radical prostatectomy compared with simple or radical laparoscopic nephrectomy. For laparoscopic pyeloplasty the difference was statistically insignificant.

The time of set up was also analysed (Tab. 1). Overall set up time was greatest for laparoscopic radical cystectomy ([E-Arm] $6.8 \pm 2.3 ; \quad$ [C-Arm] $7.1 \pm 1.9 \mathrm{~min}$ ) and least for pyeloplasty ([E-Arm] $5.1 \pm 1.8$; [C-Arm] $5.3 \pm 1.7 \mathrm{~min}$ ) and there was no statistically significant difference between set up times for the E-Arm and C-Arm groups. The set-up time for all cases was under $8 \mathrm{~min}$. Use of the EndoAssist device has no effect on set up time compared with the conventional approach. With regard to surgical performance, all three surgeons reported that the EndoAssist device did not compromise surgical performance. They also reported that the EndoAssist device was a viable option which enabled optimum task performance for all the types of case studied, and comparable with use of a human camera driver. There were no significant differences between complication rates or total operative time for procedures conducted with the EndoAssist device or with a conventional human assistant.

With regard to the need to clean the scope, we found this was not a useful tool for measuring the performance of the two arms because it varies from case to case. Scope cleaning depends on several factors, e.g. the assistant driving the camera, the body fat of the patient, the type of surgery being performed, and patient anatomy.

\section{Discussion}

The realization that the camera holder need not necessarily be a human and that a given laparoscopic surgical task could be conducted by devices under the 
Table 1 Setting up times for EndoAssist and for the conventional human driver template (mean time in minute \pm standard deviation)

\begin{tabular}{|c|c|c|c|c|}
\hline Procedure & $\begin{array}{l}\text { Total number of cases } \\
\text { using endoassist }[\mathrm{E}]\end{array}$ & $\begin{array}{l}\text { Total number of cases } \\
\text { conventional }[\mathrm{C}]\end{array}$ & Mean setting up time (mins) & $\begin{array}{l}\text { Statistically } \\
\text { significant }[\mathrm{Y} / \mathrm{N}]\end{array}$ \\
\hline Nephrectomy & 16 & 17 & {$[\mathrm{E}] 5.9 \pm 1.2 \mathrm{Vs}[\mathrm{C}] 5.6 \pm 1.3$} & $\mathrm{~N}$ \\
\hline Pyeloplasty & 4 & 4 & [E] $5.1 \pm 1.8 \mathrm{Vs}$ [C] $5.3 \pm 1.7$ & $\mathrm{~N}$ \\
\hline Prostatectomy & 3 & 3 & [E] $5.8 \pm 2.8 \mathrm{Vs}[\mathrm{C}] 5.6 \pm 2.9$ & $\mathrm{~N}$ \\
\hline Cyctectomy & 2 & 2 & [E] $6.8 \pm 2.3 \mathrm{Vs}[\mathrm{C}] 7.1 \pm 1.9$ & $\mathrm{~N}$ \\
\hline
\end{tabular}

direct or indirect control of the operating surgeon has led to the objective and subjective evaluation of several devices when undertaking laparoscopic urological surgery. Kavoussi et al. [7], in 1995 reported results of a study on the accuracy and use of a robotic surgical arm compared with a human surgical assistant during urological laparoscopic surgery. They concluded that camera positioning was significantly steadier with fewer inadvertent movements when under robotic rather than human control. They found no significant difference in the operative times during dissections using the robot or human assistant, however. The same team [6] later published their findings on the use of surgeon-controlled robotic arms as a substitute for human assistants. They found that simultaneous use of remote-controlled robotic arms as surgical assistants is feasible in laparoscopic urological surgery. They also found operating time did not increase when the robotic arms were used and there was no difference between set-up and breakdown times in their series of 17 laparoscopic urological cases. Exploration of alternatives to human camera holders was not confined to urologists alone; in the same year non-urological surgeons experimented with similar concepts. Begin et al. [8], for example, defined the motions of the human camera operator and expressed them mathematically by use of a spherical displacement model. They then applied this to a revolving robotic arm with six degrees of freedom in conjunction an automated camera in the performance of cholecystectomy and other procedures in animal models. Geis et al. [9] soon looked at robotic arm enhancement and its effect on efficiency and as a means of reducing resource use in complex minimally invasive surgical procedures. They used robotic arm enhancement to minimize resource and personnel use during minimally invasive procedures. They concluded that robotic arm enhancement reduced costs and minimized risk for patients undergoing minimally invasive surgical procedures. They also found, in their general surgical series, that safety, versatility, and diminished use of resources had an overall benefit. With cost containment in mind, Turner compared the cost-effectiveness of using a robotic assistant instead of a human assistant in a series of 12 cases of solo surgery in laparoscopic bladder neck suspension. He concluded that the cost of the robotic arm was less than that of human systems and that the former was a cost-effective means of performing the procedure [10]. In a comparative analysis of several studies to determine whether the robotic arm can effectively provide the surgeon with complete control of the surgical field, and the impact of this device on overall cost, Dunlap et al. [11] found that a robotic arm not only outperformed human camera holders but also improved efficiency and cost savings. The current price of the EndoAssist device is just under $\$ 100,000$ US dollars, which is similar to that of an AESOP device. These costs when balanced against use of man power and cost per hour of employing a human camera driver points in favour of the non-human-controlled camera devices from a strictly health economics point of view.

Having discovered that non-human-controlled camera devices were economically and technically feasible, several groups sought to compare the different devices. Allaf et al. [1] evaluated the standard foot pedal for the AESOP robot compared with a voice control interface. They concluded that voice control was more accurate, and had the advantage of not requiring the surgeon to look away from the operative field, but that it was slower and required more attention as an interface. Wagner et al. [12] were the first to directly compare the EndoAssist and AESOP, using the index procedure of laparoscopic radical prostatectomy. After analysis of prospectively collected data for 20 patients they concluded that the EndoAssist was as efficient as AESOP with regard to surgical performance and that the advantages of the EndoAssist included its accurate response and its ability to provide the surgeon with complete control of the desired operative view without relying on an assistant. In contrast, the disadvantages were cited as its large size, the inability to mount it on the table, and its dependence on pedal activation. A review of published literature revealed that the advantage of the EndoAssist over other camera-holding robots seems to be its short response time and the ability for multiple surgeons to be trained in the use of the same robot without the need to generate different sound cards for each user. 
Perhaps most importantly, there is complete autonomy of the surgeon to obtain the desired optimum operator view without relying on the experience and skill of his assistant. The disadvantages of the EndoAssist seem to be its fairly large footprint (it cannot be mounted on the operating table) and that use of the foot-operated clutch sometimes results in the need to take one's eye off the operative field to search for the foot pedal. This skill is difficult to master and usually comes only by assisting the same surgeon over several procedures. The camera operator is usually a trainee who is much less experienced than the operating surgeon and, therefore, the difficulty is compounded. Should retroperitoneoscopy be adopted for kidney surgery, this also necessitates placement of the ports while the patient is in the flank position. As a result, the camera operator must hold the position of the camera in an ergonomic and uncomfortable way for a significant length of time.

From our series of 25 cases using the EndoAssist and 26 conventional laparoscopic cases we made several interesting observations. Several advantages of the EndoAssist are immediately apparent, primarily the intuitive positioning of the camera by the surgeon to optimise his operating field and the potential reduction in cost if no assistant is required.

It is necessary to learn to use the equipment, but proficiency in the execution of the robotic movements is easily acquired in a few minutes. There was no neck or shoulder discomfort, because the head-mounted sensor weighs less than $10 \mathrm{~g}$ and can easily be mounted on to a headband should the surgeon so decide. The BPDS revealed that neither procedure resulted in more discomfort. The EndoAssist enabled the surgeon to intuitively control his field of laparoscopic vision while coordinating the movements of his instrumentation.

\section{Conclusion}

The EndoAssist is an effective, easy to use device for robotic camera driving which reduces the constraint of requiring an experienced camera driver for optimum visualisation during laparoscopic urological procedures. Further large-scale feasibility studies including health economic analyses are warranted.

\section{References}

1. Allaf ME, Jackman SV, Schulam PG, Cadeddu JA, Lee BR, Moore RG, Kavoussi LR (1998) Laparoscopic visual field. Voice vs foot pedal interfaces for control of the AESOP robot. Surg Endosc 12(12):1415-1418

2. Nebot PB, Jain Y, Haylett K, Stone R, McCloy R (2003) Comparison of task performance of the camera-holder robots EndoAssist and Aesop. Surg Laparosc Endosc Percutan Tech 13(5):334-338

3. Aiono S, Gilbert JM, Soin B, Finlay PA, Gordan A (2002) Controlled trial of the introduction of a robotic camera assistant (EndoAssist) for laparoscopic cholecystectomy. Surg Endosc 16(9):1267-1270. Epub 2002 Jun 14

4. Sackier JM, Wang Y. (1994) Robotically assisted laparoscopic surgery. From concept to development. Surg Endosc 8(1):63-66

5. Su LM, Link RE, Bhayani SB, Sullivan W, Pavlovich CP (2004) Nerve-sparing laparoscopic radical prostatectomy: replicating the open surgical technique. Urology 64(1):123127

6. Partin AW, Adams JB, Moore RG, Kavoussi LR (1995) Complete robot-assisted laparoscopic urologic surgery: a preliminary report. J Am Coll Surg 181(6):552-557

7. Kavoussi LR, Moore RG, Adams JB, Partin AW (1995) Comparison of robotic versus human laparoscopic camera control. J Urol 154(6):2134-2136. Erratum in: J Urol 1997 158(4):1530

8. Begin E, Gagner M, Hurteau R, de Santis S, Pomp A (1995) A robotic camera for laparoscopic surgery: conception and experimental results. Surg Laparosc Endosc 5(1):6-11

9. Geis WP, Kim HC, Brennan EJ Jr, McAfee PC, Wang Y (1996) Robotic arm enhancement to accommodate improved efficiency and decreased resource utilization in complex minimally invasive surgical procedures. Stud Health Technol Inform 29:471-481

10. Turner DJ (1996) Solo surgery-with the aid of a robotic assistant. J Telemed Telecare 2(Suppl 1):46-48

11. Dunlap KD, Wanzer L (1998) Is the robotic arm a costeffective surgical tool? AORN J 68(2):265-272. Review. Erratum in: AORN J 68(3):368

12. Wagner AA, Varkarakis IM, Link RE, Sullivan W, Su LM (2006) Comparison of surgical performance during laparoscopic radical prostatectomy of two robotic camera holders, EndoAssist and AESOP: a pilot study. Urology 68(1):70-74 